\title{
Lifestyle Medicine and Psychological Well-Being toward Health Promotion: A Cross-Sectional Study on Palermo (Southern Italy) Undergraduates
}

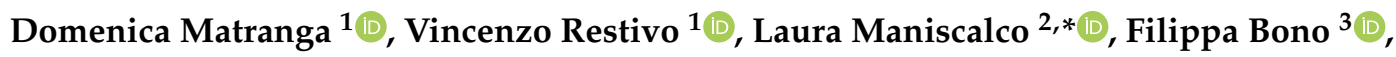 \\ Giuseppe Pizzo $^{4}{ }^{\mathbb{D}}$, Giuseppe Lanza ${ }^{1}$, Valerio Gaglio ${ }^{1}$, Walter Mazzucco ${ }^{1}{ }^{1}$ and Silvana Miceli ${ }^{5}$ \\ 1 Department of Health Promotion, Mother and Infant Care, Internal and Specialized Medicine \\ "G. D'Alessandro", University of Palermo, 90127 Palermo, Italy; domenica.matranga@unipa.it (D.M.); \\ vincenzo.restivo@unipa.it (V.R.); giuseppeluigimarco.lanza@you.unipa.it (G.L.); \\ valerio.gaglio@unipa.it (V.G.); walter.mazzucco@unipa.it (W.M.) \\ 2 Department of Biomedicine, Neuroscience and Advanced Diagnostics, University of Palermo, \\ 90127 Palermo, Italy \\ 3 Department of Economics, Business and Statistics, University of Palermo, 90128 Palermo, Italy; \\ filippa.bono@unipa.it \\ 4 Department of Surgical, Oncological and Oral Sciences, University of Palermo, 90127 Palermo, Italy; \\ giuseppe.pizzo@unipa.it \\ 5 Department of Psychology, Educational Science and Human Movement, University of Palermo, \\ 90128 Palermo, Italy; silvana.miceli56@unipa.it \\ * Correspondence: laura.maniscalco04@unipa.it
}

Received: 6 July 2020; Accepted: 23 July 2020; Published: 28 July 2020

\begin{abstract}
Aim: To assess the attitude toward Lifestyle Medicine and healthy behaviours among students in the healthcare area and to demonstrate its association to psychological well-being; (2) Methods: A cross-sectional study is conducted among 508 undergraduates of the University of Palermo (140 (27.6\%) in the healthcare area and $368(72.4 \%)$ in the non-healthcare area), during the academic year 2018-2019. Psychological well-being is measured through two dimensions of eudaimonia and hedonia, using the 10-item Hedonic and Eudaimonic Motives for Activities-Revised (HEMA-R) scale, with answers coded on a 7-point scale. The association between demographic and modifiable behavioural risk factors for chronic diseases is assessed through crude and adjusted Odds ratios with 95\% confidence intervals; (3) Results: Orientation to both hedonia and eudaimonia is significantly associated to the Mediterranean diet (ORAdj $=2.28 ; 95 \% \mathrm{CI}=(1.42-3.70)$ ) and drinking spirits less than once a week (ORAdj $=1.89 ; 95 \% \mathrm{CI}=(1.10-3.27))$ and once a week or more (ORAdj $=6.02 ; 95 \% \mathrm{CI}=(1.05-34.52))$, while these conditions occur together less frequently for current smokers (ORAdj $=0.38 ; 95 \% \mathrm{CI}=(0.18-0.81)$ ). Students inclined to well-being consider healthcare professionals as models for their patients and all people in general $(\mathrm{OR}=1.96,95 \% \mathrm{CI}=(1.28-3.00))$; (4) Conclusions: The positive relation found between a virtuous lifestyle and psychological well-being suggests the construction, development and cultivation of individual skills are a means to succeed in counteracting at risk behaviours for health.
\end{abstract}

Keywords: lifestyle medicine; chronic diseases; modifiable behaviours; risk factors; psychological well-being; public health; eudaimonia; hedonia 


\section{Introduction}

Worldwide, countries put policy strategies and health programs in place to counteract the spread chronic diseases and to promote healthy behaviours [1]. Poor diet, physical inactivity, tobacco, and alcohol abuse are considered the modifiable behavioural risk factors (MBRF) determining the greatest burden of chronic diseases [2]. The World Health Organization [1] reports that tobacco accounts for over 7.2 million deaths every year, including those related to being exposed to passive smoke, and is expected to increase markedly over the coming years. It also estimates that excess salt/sodium intake is responsible for 4.1 million annual deaths and that insufficient physical activity accounts for 1.6 million deaths annually. Further, nearly 3 million annual deaths are attributable to alcohol abuse (5.3\% of all deaths) [1]. To support the change of lifestyle as a first line of prevention, the Lifestyle Medicine (LM) approach, as defined in 2010 by the Journal of the American Medical Association, is "the evidence-based practice of assisting individuals and families to adopt and sustain behaviours that can improve health and quality of life" [3]. The American College of Lifestyle Medicine (ACLM) establishes six ways to take control of health through regular physical exercise, adequate and good quality sleep, cessation of smoking, stress management and maintenance of relationships. Moreover, LM advocates for a whole-food, plant-based diet for a nutritional lifestyle that is based on whole, minimally processed foods, plants, including vegetables, fruits, whole grains, legumes, seeds and nuts, and little or no intake of animal products. Additionally, there is a preference for quality or organic food and zero-miles products. Physicians are the first source of information about health and healthy behaviours for their patients [4] and physician counselling has been demonstrated to be effective for weight loss [5], smoke cessation [6] and alcohol intake [7]. Regarding the skills required to practice LM, primary care physicians are called to promote healthy behaviours as the basis of medical care, disease prevention and health promotion, as well as providing the knowledge of the mechanisms by which specific lifestyle changes can have a positive effect on patient health outcomes [8]. Considering this, it is essential to involve medical students in shaping and acquiring these skills through education and the alignment of medical curricula. Medical students report a lack of knowledge and poor skills to counsel their patients to improve lifestyle behaviours [9]. Even if the need to steer medical students to disease prevention and health promotion has been acknowledged, it must be noted that medical curricula are devoid of some content, such as positive psychology and psychological well-being [10], which are needed to perform counselling effectively [11].

Individuals experiencing a state of well-being are less likely to report alcohol and drug addiction, be on a poor diet or be sedentary [12], and to develop and maintain better social relationships [13]. Recently, the interest in well-being within the psychological field has grown rapidly to investigate the sources of happiness and the many facets of human flourishing [14]. Taking this perspective, we can distinguish two different approaches: "hedonia" that focuses on pleasure, happiness and the achievement of well-being through the satisfaction of one's desires [15] and "eudaimonia", according to which well-being is obtained by fulfilling one's potential in the pursuit of complex and meaningful goals [16]. According to Huta [17], the eudaimonic orientation is defined in terms of four core elements: authenticity, meaning, excellence and growth. The first refers to the possibility of living and acting in accordance with one's values and one's self; the second concerns the ability to identify the meaning of things and their value; the third refers to the struggle of the human being to reach high standards of ethical behaviour; and the fourth, finally, concerns the ability to actualise one's potential. Consequently, the eudaimonic vision is based on the concept of flourishing, true self, actualising potential, meaning, objectives, purposes, and personal expression and implies a process of continuous construction, development, and cultivation of individual skills. To contrast, the author defines the hedonic orientation as the pursuit of two elements: pleasure, that is the search for pleasant sensations and emotions; and comfort, the tendency to seek easy and painless solutions. A hedonic orientation, therefore, pushes the individual in search of what is subjectively pleasant [17]. 
Taking the perspective of medical education, the positive relation between a virtuous lifestyle and psychological well-being suggests promoting the development of curricula where a varied spectrum of LM topics, such as nutrition and exercise together with the analysis of the underlying psychological processes, are provided and developed in an integrated manner [18].

To assess the attitude toward LM and lifestyle behaviours among students in the healthcare area, and to demonstrate the association with psychological well-being, we conduct a cross-sectional study among undergraduates of the University of Palermo during the academic year 2018-2019. More in depth, our study assumes that the adoption of healthy behaviours could be significantly associated with psychological well-being.

\section{Materials and Methods}

\subsection{Study Design}

A cross-sectional study was designed to investigate the study aims on a sample of students attending health (medicine, dentistry, and other health professional courses) and non-health (economics and psychological science) degree courses. To this end, a questionnaire articulated into five sections (demographics, diet habits, smoking, physical activity, and psychological well-being) was structured. To assess the lifestyle, composite indicators were constructed for (i) eating habits, (ii) alcohol consumption, (iii) sedentary lifestyle, (iv) smoking, (v) sexual habits, and (vi) addictions. The diet habits section included questions regarding variations which occurred in the last two and five years in the respondent's dietary habits, the food frequency test, and questions about alcohol intake [19], and binge drinking. The most widespread healthy diet in Italy is the Mediterranean diet that consists of "a high intake of vegetables, legumes, fruits and nuts, and cereals, and a high intake of olive oil but a low intake of saturated lipids, a moderately high intake of fish, a low-to-moderate intake of dairy products, a low intake of meat and poultry, and a regular but moderate intake of ethanol, primarily in the form of wine and generally during meals" [20]. Following Trichopoulou et al. [19], for subjects whose consumption was adherent to the Mediterranean diet, a score from 0 (minimum adherence) to 10 (maximum adherence) was assigned for each respondent and the median value was chosen as a cut-off to define the respondent's adherence to the Mediterranean diet (adherent if the score $>$ median and not adherent if the score $\leq$ median). Alcohol consumption was assessed concerning wine, beer and spirits and expressed through three frequency categories (never, less than once a week, once a week or more).

Binge drinking typically happens when men consume 5 or more drinks or women consume 4 or more drinks in about $2 \mathrm{~h}$. Binging was detected through a question about the occurrence of a binge episode in the last 12 months and expressed as a binary variable with "yes" or "no" response categories [21].

The smoking section was developed in agreement with the WHO guidelines about tobacco use. Smoking status was expressed as a categorical variable with three categories, non-smoker, former and current smoker [1]. Physical activity was defined as a binary variable with "yes" or "no" as the response categories [22].

The measure used to assess eudaimonic and hedonic orientations is the Hedonic and Eudaimonic Motives for Activities-Revised (HEMA-R) scale, which represents a revision of the original Hema scale [23]. The scale is made up of 10 items, five of which refer to eudaimonic orientation and five to hedonic orientation. Particularly, hedonia is expressed through the concepts of "pleasure" and "comfort". Conversely, eudaimonia is demonstrated through the concepts of "authenticity", "excellence", "growth" and "meaning". The subjects were asked to evaluate the degree to which they usually carry out their activities according to the intentions indicated and independently of the achievement of the objective. Participants gave ratings on various eudaimonic and hedonic motives, which were intermixed on a scale from 1 (not at all) to 7 (very much). A subject was considered oriented to hedonia or eudaimonia if the respective score was not less than the median. Well-being was 
expressed through a categorical variable with four categories (no orientation, hedoniac, eudaimoniac, both orientations).

To assess each student's health, each subject was asked if he/she had ever suffered from diabetes, hypertension, hypercholesterolemia, other cardiovascular diseases, neoplasia, or other chronic diseases. The occurrence of chronic disease was expressed as a binary variable with category "yes" in the case of at least one chronic disease and "no" in the case of none.

Finally, the questionnaire included three specific questions about the role of healthcare professionals for prevention and health promotion. First, each participant was asked if healthcare professionals could be considered as models for their patients and people in general; second, if they should regularly counsel their patients for tobacco cessation and, third, if a patient has an increased probability to stop smoking if assisted by his/her practitioner or nurse. Regarding all questions, the possible answer categories were "yes" and "no".

The study was presented by the research team during a seminar entitled "Communicative skills for the promotion of correct lifestyles and the prevention of chronic degenerative diseases" opened to students from different study areas. Then, students were invited to adhere to the survey by self-administering the questionnaire through the Google platform. The study was approved by the Ethical Committee of the "Azienda Universitaria Policlinico Paolo Giaccone" of Palermo (Reference number 08/2018).

\subsection{Statistical Methods}

Categorical variables were expressed as counts and percentages, and continuous variables were categorized using the median as the cut-off value. Univariable association between demographic and Modifiable Behavioural Risk Factors (MBRFs) was assessed using the Chi-square test or the Fisher exact test, as appropriate, and measured through crude Odds Ratios (OR) and 95\% Confidence Intervals (CIs). Multinomial logistic regression was performed to assess the likelihood of being oriented toward eudaimonia, hedonia or both, compared to no orientation in relation to demographics and MBRFs that had significant results using univariable analysis. Results of multivariable analysis were expressed as adjusted ORs (AdjOR) and 95\% CIs.

Stata IC/15.1 (StataCorp LLC, Texas, TX, USA) was used for statistical analysis, and a $p$-value $<0.05$ was chosen as the statistical significance cut-off.

\section{Results}

A sample of 508 undergraduate students, $140(27.6 \%)$ from the health area and $368(72.4 \%)$ from the non-health area, was recruited during the study. The majority of the respondents attended the first year of study $(\mathrm{n}=348 ; 68.5 \%)$, while the remaining part was divided between the second and third attendance year, $81(15.9 \%)$ and $79(15.6 \%)$, respectively.

The Hedonic and Eudaimonic Motives for Activities-Revised (HEMA-R) was available for all students, $325(64.0 \%)$ females, with parents highly educated or graduated $(79 \%$ fathers and $81 \%$ mothers), mostly on-site students $(267,43.8 \%)$ followed by off-site $(142,28 \%)$ and commuter $(99,15.5 \%)$ students. The attitude to be hedonic was lower for females than for males (Odds Ratios $(\mathrm{OR})=0.56$, $95 \%$ Confidence Intervals $(C I)=(0.35-0.91))$. Notably, there was not any significant difference between students in the healthcare and non-healthcare area to be more inclined to either hedonia or eudaimonia (Table 1). 
Using univariable analysis, students following the Mediterranean diet were more inclined to eudaimonia ( $\mathrm{OR}=2.50,95 \% \mathrm{CI}=[1.52-4.12])$, while those with binge experience in the past were less prone $(\mathrm{OR}=0.48,95 \% \mathrm{CI}=[0.29-0.79])$. Overweight students were more prone to hedonia than normal weight ones $(\mathrm{OR}=2.33,95 \% \mathrm{CI}=[1.11-4.95])$, as well as students drinking beer less than once a week $(\mathrm{OR}=2.15,95 \% \mathrm{CI}=[1.14-4.05])$ and once a week or more $(\mathrm{OR}=2.81,95 \% \mathrm{CI}=[1.23-6.42])$, and for students reporting taking spirits sometimes $(\mathrm{OR}=2.01,95 \% \mathrm{CI}=[1.17-3.46])$. Furthermore, there was a statistically significant association with experiencing binge drinking (OR $=1.6295 \% \mathrm{CI}=[1.00-2.62])$. Students accustomed to tobacco were less likely to be oriented to both hedonia and eudaimonia $(\mathrm{OR}=0.4795 \% \mathrm{CI}=[0.28-0.79]$ for former smokers and $\mathrm{OR}=0.4095 \% \mathrm{CI}=[0.22-0.75]$ for current smokers). Conversely, students drinking spirits less than once a week $(\mathrm{OR}=1.5095 \% \mathrm{CI}=[1.00-2.24])$ and once a week or more $(\mathrm{OR}=4.2995 \% \mathrm{CI}=[1.14-16.17])$ and those reporting binging experiences $(\mathrm{OR}=1.4395 \% \mathrm{CI}=[0.98-2.09])$ were more oriented to both dimensions of psychological well-being (Table 2).

Students inclined to both well-being dimensions considered healthcare professionals as models for their patients and all people in general (OR $=1.96,95 \% \mathrm{CI}=[1.28-3.00])$. Students in the healthcare area were more inclined than those in non-healthcare fields to consider that healthcare professionals should counsel their patients for tobacco cessation on a regular basis $(\mathrm{OR}=8.03$; 95\% CI $=[1.05-61.3])$ (Table 3).

Using multivariable analysis, the Mediterranean diet was confirmed as the only one statistically significant for being eudaimonic (AdjOR $=3.27 ; 96 \% \mathrm{CI}=[1.83-5.87]$ ). Additionally, experiencing both hedonia and eudaimonia was significantly associated to the Mediterranean diet (AdjOR $=2.28$; $95 \% \mathrm{CI}=[1.42-3.70])$ as well as drinking spirits less than once a week $(\mathrm{AdORj}=1.89 ; 95 \% \mathrm{CI}=[1.10-3.27])$, while these conditions occurred together less frequently for current smokers (AdjOR $=0.38$; $95 \%$ CI $=[0.18-0.87]$ ) (Table 4). 
Table 1. Psychological well-being by socio-demographic and health characteristics in a sample of 508 undergraduates of University of Palermo: univariable analysis.

\begin{tabular}{|c|c|c|c|c|c|c|c|c|c|c|}
\hline \multirow{2}{*}{ Characteristics $\S$} & \multirow[b]{2}{*}{$\mathbf{N}$} & \multicolumn{3}{|c|}{ Eudaimonia } & \multicolumn{3}{|c|}{ Hedonia } & \multicolumn{3}{|c|}{ Both } \\
\hline & & n $(\%)$ & OR $(95 \% C I) \S \S$ & $p$-Value & n $(\%)$ & OR $(95 \% C I) \S \S$ & $p$-Value & n $(\%)$ & OR $(95 \% C I) \S \S$ & $p$-Value \\
\hline \multicolumn{11}{|l|}{ age (ys) } \\
\hline$\leq 20$ & 340 & $54(15.88)$ & 1.00 & 0.639 & $58(17.06)$ & & 0.425 & $101(29.71)$ & 1 & 0.171 \\
\hline$>20$ & 168 & $24(14.29)$ & $0.88(0.52-1.49)$ & & $24(14.29)$ & $0.81(0.48-1.36)$ & & $60(35.71)$ & $1.31(0.89-1.95)$ & \\
\hline \multicolumn{11}{|l|}{ gender } \\
\hline male & 183 & $23(12.57)$ & 1 & 0.192 & $39(21.31)$ & 1 & 0.018 & $63(34.43)$ & 1.00 & 0.321 \\
\hline female & 325 & $55(16.92)$ & $1.42(0.84-2.40)$ & & $43(13.23)$ & $0.56(0.35-0.91)$ & & $98(30.15)$ & $0.82(0.56-1.21)$ & \\
\hline \multicolumn{11}{|l|}{ chronic diseases } \\
\hline no & 491 & $73(14.87)$ & 1 & 0.102 & $81(16.50)$ & 1 & 0.243 & $157(31.98)$ & 1 & 0.462 \\
\hline yes & 17 & $55(29.41)$ & $2.39(0.81-7.00)$ & & $1(5.88)$ & $0.32(0.04-2.43)$ & & $4(23.53)$ & $0.65(0.21-2.04)$ & \\
\hline \multicolumn{11}{|l|}{ year of study } \\
\hline first & 348 & $55(15.80)$ & 1 & & $56(16.09)$ & 1 & & 109 (31.32) & 1 & \\
\hline second & 81 & $9(11.11)$ & $0.67(0.31-1.40)$ & 0.286 & $15(18.52)$ & $1.19(0.63-2.23)$ & 0.597 & $29(35.80)$ & $1.22(0.74-2.03)$ & 0.437 \\
\hline third & 79 & $14(17.72)$ & $1.15(0.60-2.19)$ & 0.676 & $11(13.92)$ & $0.84(0.42-1.70)$ & 0.633 & $23(29.11)$ & $0.90(0.53-1.54)$ & 0.702 \\
\hline \multicolumn{11}{|l|}{ father education } \\
\hline primary & 106 & $14(13.21)$ & 1 & & $14(13.21)$ & 1 & & $35(33.02)$ & 1 & \\
\hline high & 203 & $34(16.75)$ & $1.32(0.67-2.59)$ & 0.415 & $36(17.73)$ & $1.42(0.72-2.77)$ & 0.306 & $57(28.08)$ & $0.79(0.48-1.32)$ & 0.368 \\
\hline graduate or higher & 198 & $30(15.15)$ & $1.17(0.59-2.33)$ & 0.647 & $32(16.16)$ & $1.27(0.64-2.50)$ & 0.494 & $68(34.34)$ & $1.06(0.64-1.75)$ & 0.816 \\
\hline \multicolumn{11}{|l|}{ mother education } \\
\hline primary & 96 & $13(13.54)$ & 1 & & $16(16.67)$ & 1 & & $34(35.02)$ & 1 & \\
\hline high & 237 & $32(13.50)$ & $1.00(0.50-2.00)$ & 0.992 & $38(16.03)$ & $0.95(0.50-1.81)$ & 0.887 & $74(31.22)$ & $0.83(0.50-1.37)$ & 0.460 \\
\hline graduate or higher & 174 & $33(18.97)$ & $1.49(0.74-3.01)$ & 0.257 & $28(16.09)$ & $0.96(0.49-1.88)$ & 0.903 & $52(29.89)$ & $0.78(0.46-1.32)$ & 0.351 \\
\hline \multicolumn{11}{|l|}{ type of student residency } \\
\hline on-site & 267 & $33(12.36)$ & 1 & & $51(19.1)$ & 1 & & $84(31.46)$ & 1 & \\
\hline off-site & 142 & $28(19.72)$ & $1.74(1.00-3.03)$ & 0.047 & 19 (13.39) & $0.65(0.37-1.16)$ & 0.144 & $48(33.80)$ & $1.11(0.72-1.72)$ & 0.630 \\
\hline commuter & 99 & 17 (17.17) & $1.47(0.78-2.79)$ & 0.234 & $12(12.12)$ & $0.58(0.30-1.15)$ & 0.117 & $29(29.29)$ & $0.90(0.54-1.50)$ & 0.691 \\
\hline \multicolumn{11}{|l|}{ type of course } \\
\hline non-healthcare area & 368 & $52(14.13)$ & 1 & 0.215 & $59(16.03)$ & 1 & 0.914 & $118(32.07)$ & 1 & 0.770 \\
\hline healthcare area & 140 & $26(18.57)$ & $1.39(0.83-2.33)$ & & $23(16.43)$ & $1.03(0.61-1.74)$ & & $43(30.71)$ & $0.94(0.62-1.43)$ & \\
\hline
\end{tabular}

$\S$ Statistics were calculated on available data; Bold values are statistically significant; §§ OR: Odds ratio; CI: Confidence Interval. 
Table 2. Psychological well-being by behavioral characteristics in a sample of 508 undergraduates of University of Palermo: univariable analysis.

\begin{tabular}{|c|c|c|c|c|c|c|c|c|c|c|}
\hline \multirow{2}{*}{ Characteristics $\S$} & \multirow[b]{2}{*}{$\mathbf{N}$} & \multicolumn{3}{|c|}{ Eudaimonia } & \multicolumn{3}{|c|}{ Hedonia } & \multicolumn{3}{|c|}{ Both } \\
\hline & & n (\%) & OR $(95 \% \mathrm{CI}) \S \S$ & $p$-Value & n (\%) & OR $(95 \% \mathrm{CI}) \S \S$ & $p$-Value & n (\%) & OR $(95 \% \mathrm{CI}) \S \S$ & $p$-Value \\
\hline $\begin{array}{c}\text { in the last } 5 \text { years, did } \\
\text { you change your weight? } \\
\text { no } \\
\text { yes }\end{array}$ & $\begin{array}{l}119 \\
389\end{array}$ & $\begin{array}{l}21(17.65) \\
57(14.65)\end{array}$ & $\begin{array}{c}1 \\
0.80(0.46-1.39)\end{array}$ & 0.428 & $\begin{array}{l}15(12.61) \\
67(17.22)\end{array}$ & $\begin{array}{c}1 \\
1.44(0.79-2.64)\end{array}$ & 0.231 & $\begin{array}{c}34(28.57) \\
127(32.65)\end{array}$ & $\begin{array}{c}1 \\
1.21(0.77-1.90)\end{array}$ & 0.403 \\
\hline $\begin{array}{c}\text { in this month, are you on } \\
\text { a diet? } \\
\text { no } \\
\text { yes }\end{array}$ & $\begin{array}{l}387 \\
121\end{array}$ & $\begin{array}{l}58(14.99) \\
20(16.53)\end{array}$ & $\begin{array}{c}1 \\
1.12(0.64-1.96)\end{array}$ & 0.682 & $\begin{array}{l}63(16.28) \\
19(15.70)\end{array}$ & $\begin{array}{c}1 \\
0.96(0.55-1.68)\end{array}$ & 0.881 & $\begin{array}{c}121(31.27) \\
40(33.06)\end{array}$ & $\begin{array}{c}1 \\
1.09(0.70-1.68)\end{array}$ & 0.712 \\
\hline $\begin{array}{c}\text { adherence to } \\
\text { Mediterranean diet }{ }^{2} \\
\text { no } \\
\text { yes }\end{array}$ & $\begin{array}{l}305 \\
203\end{array}$ & $\begin{array}{l}32(10.49) \\
46(22.66)\end{array}$ & $\begin{array}{c}1.00 \\
2.50(1.52-4.12)\end{array}$ & $<0.001$ & $\begin{array}{c}54(17.7) \\
28(13.79)\end{array}$ & $\begin{array}{c}1 \\
0.74(0.45-1.22)\end{array}$ & 0.241 & $\begin{array}{l}87(28.52) \\
74(36.45)\end{array}$ & $\begin{array}{c}1 . \\
1.44(0.98-2.10)\end{array}$ & 0.060 \\
\hline $\begin{array}{l}\text { weight status } \\
\text { normal } \\
\text { overweight } \\
\text { obese }\end{array}$ & $\begin{array}{c}262 \\
47 \\
50\end{array}$ & $\begin{array}{l}51(19.47) \\
7(14.89) \\
7(14.00)\end{array}$ & $\begin{array}{c}1.00 \\
0.72(0.31-1.71) \\
0.67(0.29-1.59)\end{array}$ & $\begin{array}{l}0.461 \\
0.363\end{array}$ & $\begin{array}{c}37(14.12) \\
13(27.66) \\
5(10.00)\end{array}$ & $\begin{array}{c}1 \\
\mathbf{2 . 3 3}(\mathbf{1 . 1 1}-4.95) \\
0.68(0.25-1.82)\end{array}$ & $\begin{array}{l}\mathbf{0 . 0 2 1} \\
0.435\end{array}$ & $\begin{array}{l}87(33.21) \\
14(29.79) \\
18(36.00)\end{array}$ & $\begin{array}{c}1 \\
0.85(0.43-1.68) \\
1.13(0.60-2.13)\end{array}$ & $\begin{array}{l}0.646 \\
0.702\end{array}$ \\
\hline $\begin{array}{l}\text { smoking status } \\
\text { non-smoker } \\
\text { former smoker } \\
\text { current smoker }\end{array}$ & $\begin{array}{c}76 \\
312 \\
120\end{array}$ & $\begin{array}{c}10(13.16) \\
57(18.27) \\
11(9.17)\end{array}$ & $\begin{array}{c}1 \\
1.48(0.71-3.05) \\
0.67(0.27-1.66)\end{array}$ & $\begin{array}{l}0.291 \\
0.380\end{array}$ & $\begin{array}{l}10(13.16) \\
47(15.06) \\
25(20.83)\end{array}$ & $\begin{array}{c}1 \\
1.17(0.56-2.44) \\
1.74(0.78-3.88)\end{array}$ & $\begin{array}{l}0.674 \\
0.173\end{array}$ & $\begin{array}{l}36(47.37) \\
93(29.81) \\
32(26.67)\end{array}$ & $\begin{array}{c}1 \\
0.47(0.28-0.79) \\
0.40(0.22-0.75)\end{array}$ & $\begin{array}{l}0.004 \\
0.003\end{array}$ \\
\hline $\begin{array}{c}\text { physical activity } \\
\text { no } \\
\text { yes }\end{array}$ & $\begin{array}{l}219 \\
289\end{array}$ & $\begin{array}{l}31(14.16) \\
47(16.26)\end{array}$ & $\begin{array}{c}1 \\
1.18(0.72-1.93)\end{array}$ & 0.514 & $\begin{array}{l}31(14.16) \\
51(17.65)\end{array}$ & $\begin{array}{c}1 \\
1.30(0.80-2.11)\end{array}$ & 0.290 & $\begin{array}{l}62(28.31) \\
99(34.26)\end{array}$ & $\begin{array}{c}1 \\
1.32(0.90-1.93)\end{array}$ & 0.154 \\
\hline $\begin{array}{c}\text { drinking wine } \\
\text { never } \\
\text { less than once a week } \\
\text { once a week or more }\end{array}$ & $\begin{array}{c}153 \\
278 \\
67\end{array}$ & $\begin{array}{l}26(16.99) \\
40(14.39) \\
11(16.42)\end{array}$ & $\begin{array}{c}1 \\
0.82(0.48-1.41) \\
0.96(0.44-2.08)\end{array}$ & $\begin{array}{l}0.473 \\
0.917\end{array}$ & $\begin{array}{l}20(13.07) \\
45(16.19) \\
15(22.39)\end{array}$ & $\begin{array}{c}1 \\
1.28(0.73-2.27) \\
1.92(0.91-4.06)\end{array}$ & $\begin{array}{l}0.388 \\
0.083\end{array}$ & $\begin{array}{l}44(28.76) \\
91(32.73) \\
23(34.33)\end{array}$ & $\begin{array}{c}1 \\
1.21(0.78-1.86) \\
1.29(0.70-2.40)\end{array}$ & $\begin{array}{l}0.395 \\
0.410\end{array}$ \\
\hline
\end{tabular}


Table 2. Cont.

\begin{tabular}{|c|c|c|c|c|c|c|c|c|c|c|}
\hline \multirow{2}{*}{ Characteristics $\S$} & \multirow[b]{2}{*}{$\mathbf{N}$} & \multicolumn{3}{|c|}{ Eudaimonia } & \multicolumn{3}{|c|}{ Hedonia } & \multicolumn{3}{|c|}{ Both } \\
\hline & & n (\%) & OR $(95 \% \mathrm{CI}) \S \S$ & $p$-Value & n (\%) & OR $(95 \% C I) \S \S$ & $p$-Value & n (\%) & OR $(95 \% \mathrm{CI}) \S \S$ & $p$-Value \\
\hline \multicolumn{11}{|l|}{ drinking beer } \\
\hline never & & $26(17.45)$ & 1 & & $14(9.40)$ & 1 & & $43(28.86)$ & 1 & \\
\hline less than once a week & 149 & 45 (15.79) & $0.89(0.52-1.51)$ & 0.657 & $52(18.25)$ & $2.15(1.14-4.05))$ & 0.015 & $91(31.93)$ & $1.16(0.75-1.78)$ & 0.511 \\
\hline once a week or more & 285 & $7(11.29)$ & $0.60(0.25-1.48)$ & 0.263 & $14(22.58)$ & $2.81(1.23-6.42)$ & 0.010 & $21(33.87)$ & $1.26(0.67-2.39)$ & 0.472 \\
\hline \multicolumn{11}{|l|}{ drinking spirits } \\
\hline never & 193 & $34(17.62)$ & 1 & & $21(10.88)$ & 1 & & $50(25.91)$ & 1 & \\
\hline less than once a week & 294 & $42(14.29)$ & $0.78(0.48-1.28)$ & 0.322 & $58(19.73)$ & $2.01(1.17-3.46)$ & 0.010 & $101(34.35)$ & $1.50(1.00-2.24)$ & 0.049 \\
\hline once a week or more & 10 & $1(10.00)$ & $0.52(0.06-4.27)$ & 0.535 & $1(10.00)$ & $0.91(0.11-7.58)$ & 0.931 & $6(60.00)$ & $4.29(1.14-16.17)$ & 0.019 \\
\hline \multicolumn{11}{|l|}{ binging } \\
\hline no & 255 & $51(20.0)$ & 1 & & $33(12.94)$ & 1 & & $71(27.84)$ & 1 & \\
\hline yes & 253 & $27(10.7)$ & $0.48(0.29-0.79)$ & 0.004 & 49 (19.37) & $1.62(1.00-2.62)$ & 0.049 & $90(35.57)$ & $1.43(0.98-2.09)$ & 0.061 \\
\hline
\end{tabular}

$\S$ Statistics were calculated on available data. Bold values are statistically significant; $\$ \S$ OR: Odds ratio; CI: Confidence Interval. 
Table 3. Role of healthcare professionals for prevention and health promotion using a well-being approach and type of course in a sample of 508 undergraduates of University of Palermo.

\begin{tabular}{|c|c|c|c|c|c|c|c|c|c|}
\hline \multirow[t]{2}{*}{ Characteristics $\S$} & \multicolumn{3}{|c|}{$\begin{array}{c}\text { Do You Think that Healthcare Professionals } \\
\text { Could Be Considered as Models for Their } \\
\text { Patients and People in General? }\end{array}$} & \multicolumn{3}{|c|}{$\begin{array}{c}\text { Do You Think that Healthcare Professionals } \\
\text { Should Counsel Their Patients for Tobacco } \\
\text { Cessation on a Regular Basis? }\end{array}$} & \multicolumn{3}{|c|}{$\begin{array}{c}\text { Do You Think that a Patient Has an Increased } \\
\text { Probability to Stop Smoking, if Assisted by } \\
\text { His/Her Practitioner or Nurse? }\end{array}$} \\
\hline & $\mathbf{N}$ & n (\%) & $\begin{array}{c}\text { OR }(95 \% \mathrm{CI}) \S \S \\
p \text {-Value }\end{array}$ & $\mathbf{N}$ & n (\%) & $\begin{array}{c}\text { OR }(95 \% \mathrm{CI}) \S \S \\
p \text {-Value }\end{array}$ & $\mathbf{N}$ & n (\%) & $\begin{array}{c}\text { OR }(95 \% \mathrm{CI}) \S \S \\
p \text {-Value }\end{array}$ \\
\hline $\begin{array}{l}\text { eudaimonia } \\
\text { no }\end{array}$ & 172 & $21(12.21)$ & \multirow[b]{2}{*}{$\begin{array}{c}1.48(0.86-2.54) \\
0.152\end{array}$} & 21 & $3(14.29)$ & \multirow[b]{2}{*}{$\begin{array}{l}1.10(0.32-3.82) \\
0.884\end{array}$} & 88 & $9(10.23)$ & \multirow[b]{2}{*}{$\begin{array}{c}1.73(0.83-3.62) \\
0.141\end{array}$} \\
\hline yes & 334 & 57 (17.07) & & 485 & 75 (15.46) & & 419 & $69(16.47)$ & \\
\hline $\begin{array}{l}\text { hedonia } \\
\text { no }\end{array}$ & 172 & 34 (19.77) & \multirow[b]{2}{*}{$\begin{array}{c}0.66(0.41-1.08) \\
0.098\end{array}$} & 21 & $3(14.29)$ & \multirow[b]{2}{*}{$\begin{array}{c}1.17(0.34-4.06) \\
0.808\end{array}$} & 88 & 17 (19.32) & \multirow[b]{2}{*}{$\begin{array}{c}0.77(0.42-1.39) \\
0.379\end{array}$} \\
\hline yes & 334 & 47 (14.07) & & 485 & 79 (16.29) & & 419 & $65(15.51)$ & \\
\hline $\begin{array}{l}\text { both } \\
\text { no }\end{array}$ & 172 & 39 (22.67) & \multirow[b]{2}{*}{$\begin{array}{c}1.96(1.28-3.00) \\
0.002\end{array}$} & 21 & $9(42.86)$ & \multirow[b]{2}{*}{$\begin{array}{l}0.60(0.255-1.46) \\
0.259\end{array}$} & 88 & 29 (32.95) & \multirow[b]{2}{*}{$\begin{array}{l}0.94(0.57-1.53) \\
0.791\end{array}$} \\
\hline yes & 334 & $122(36.53)$ & & 485 & $151(31.13)$ & & 419 & $132(31.50)$ & \\
\hline $\begin{array}{l}\text { type of course } \\
\text { non-healthcare }\end{array}$ & 366 & $238(65.0)$ & \multirow[b]{2}{*}{$\begin{array}{c}1.17(0.77-1.78) \\
0.451\end{array}$} & 366 & 346 (94.5) & \multirow[b]{2}{*}{$\begin{array}{c}8.03(1.05-61.3) \\
0.013\end{array}$} & 367 & $296(80.7)$ & \multirow[b]{2}{*}{$\begin{array}{c}1.74(0.98-3.08) \\
0.056\end{array}$} \\
\hline healthcare & 140 & $96(68.6)$ & & 140 & $139(99.3)$ & & 140 & $123(87.9)$ & \\
\hline
\end{tabular}


Table 4. Multivariable analysis of well-being by modifiable behavioral characteristics in a sample of 508 undergraduates of University of Palermo.

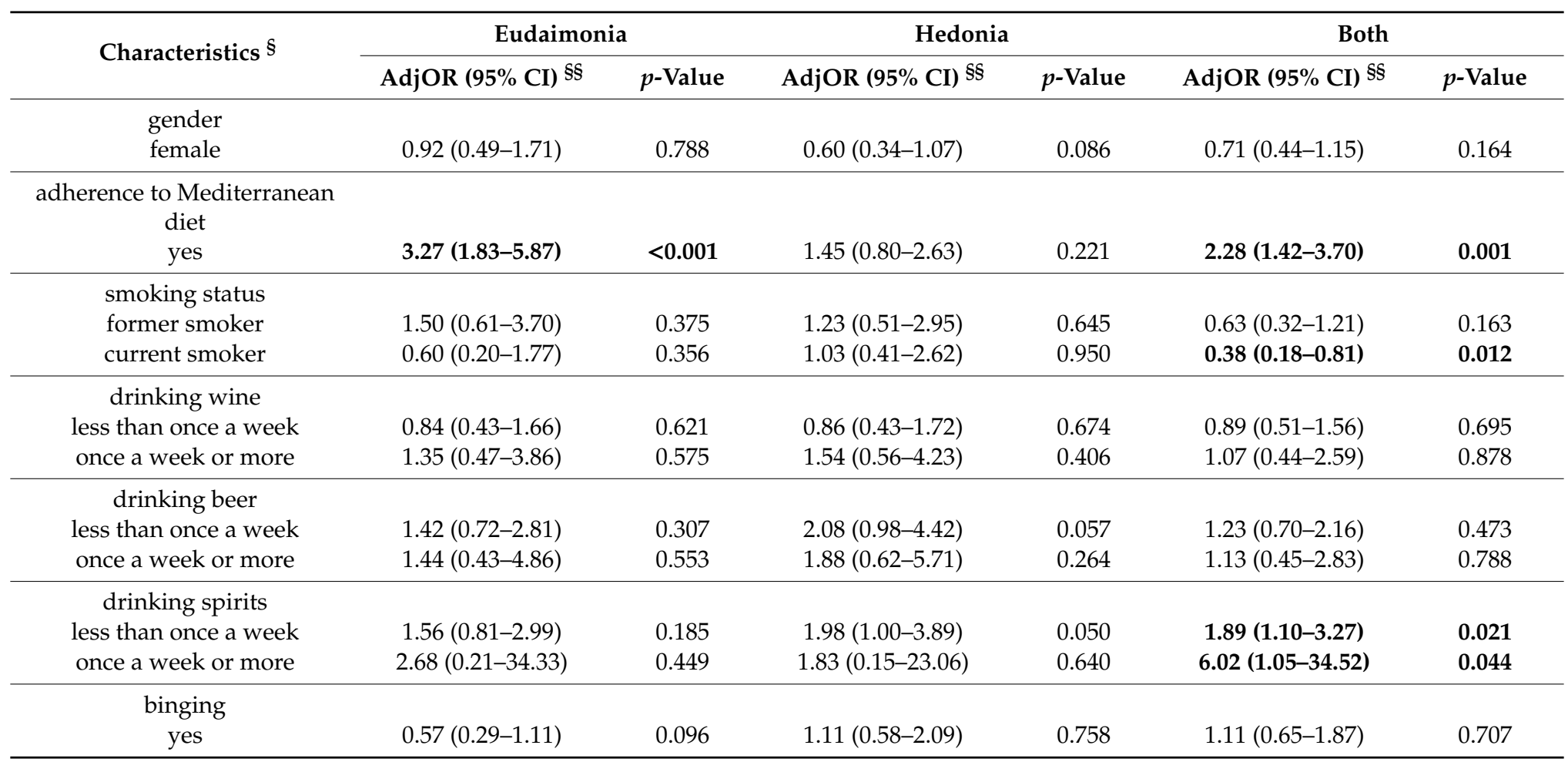

$\S$ Reference categories: "No" for Adherence to Mediterranean Diet, Physical activity, Binging, "Male" for Gender, "Non-smoker" for Smoking status, "Never" for Drinking wine, Drinking beer, Drinking spirits. Statistics were calculated on available data; Bold values are statistically significant; $\$ \S$ AdjOR: Adjusted Odds ratio; CI: Confidence Interval. 


\section{Discussion}

Lifestyle Medicine has produced significant changes in the concept of health, moving from a care-centred approach to an approach focused on promoting well-being. Our findings document a positive relationship between healthy behaviours and psychological well-being and suggest the construction, development, and cultivation of individual skills as a means to be successful in counteracting behaviours at risk for health. Specifically, we have deepened the link between eudaimonic well-being and healthy behaviours, with particular regard to nutrition, physical activity, alcohol, and tobacco abuse. Our results indicate that students adhering to the Mediterranean diet were more likely to be oriented toward psychological well-being, in line with other literature [12]. Additionally, taking alcohol less than once a week or once a week or more was significantly related to both hedonic and eudaimonic well-being. This surprising result may be explained as the occasional intake of alcohol in the young population typically occurs between meals within a social context characterized by interactions that strengthen individual identity and group cohesion [24]. Conversely, the result that current smokers are less oriented toward the hedonic and eudaimonic perspective can be explained as tobacco consumption is perceived as an addiction more than a condition of pleasure [25].

Different from the classical approaches followed by studies conducted on medical students and residents [26], our study explicitly examined the role of the eudaimonic dimension in influencing the implementation of healthy lifestyles. The results are in line with the most recent developments in the literature, which have shown that the pursuit of eudaimonic objectives undoubtedly represents a protective factor from the implementation of health risk behaviours [27].

The result that eudaimonic students showed a trend toward improvement in their own healthy behaviours is worthy of note for the effective education of future Lifestyle Medicine (LM) practitioners, as it has been demonstrated that there is a positive association between preventive healthy habits of physicians and their patients [28]. Eudaimonic subjects were found to be more prone to consider healthcare professionals as a model for the whole community and students in the healthcare field were more oriented to counselling than non-healthcare students.

Ryff [29] suggests the science of eudaimonia shows its relevance for multiple aspects of physical functioning, as well as for health research. Eudaimonic well-being is capable of leading the individual to functional and highly adaptive behaviour patterns. The assumption of a eudaimonic perspective that focuses on the subject could indirectly lead to a change in lifestyle. Unlike many coaching programs developed for the promotion of health that mainly aim to modify specific dysfunctional behaviours, other studies [30] have developed interventions whose participation induced greater subjective vitality in the subjects, leading them to develop changes in their lifestyles not explicitly requested. This is the typical approach of the LM practitioner, to steer the patient to commit oneself to achieve greater awareness of oneself and of one's life meanings, to implement virtuous and healthy behaviours. The LM practitioner has the skills to assess patient attitudes toward making healthy behaviour changes, helping them in self-managing healthy behaviours and recognising negative stress responses, also with the aid of technology and multidisciplinary teamwork. Focusing on the eudaimonic perspective, therefore, means creating a setting in which to explore oneself, understand one's potential, one's sense of life, far from the emotional stress derived from programs based on diets, exercise, or reduction in tobacco consumption, as examples. Promoting well-being, in any of its forms, therefore, can represent a useful goal to create conditions in which life is perceived as interesting, rewarding, and full of meaning.

The study did not find any significant difference between students attending courses in the healthcare and non-healthcare areas concerning the relation between lifestyle and well-being. This suggests that enhancing the awareness of one's well-being is useful for the balanced growth of all individuals, but it becomes an essential skill of valued students in the healthcare field and of effective LM practitioners. Therefore, our study encourages the development of curricula for medical students that include, in addition to topics such as nutrition and exercise, the analysis of the psychological processes underlying well-being and positive mental health. This result is in line with the most recent contents 
of the Lifestyle Medicine Core Competencies Program (https://lifestylemedicine.org/ACLM/Education/ Certification). This program adds to core competency skills, nutrition, tobacco cessation, physical activity, sleep education, alcohol use and weight management, as well as the skills related to health and wellness coaching, emotional wellness, and mindfulness. The usual strategies used by the different countries to reduce alcohol and tobacco consumption or the traditional intervention programs aimed at pushing the population toward healthy eating behaviours have not always proved effective in the long term.

There are some flaws in this study. The first one regards the recruitment of subjects. Actually, the two student groups were different as only those in the healthcare field were compelled to the seminar where the study was presented. Therefore, it can be hypothesised that students in the non-healthcare area was disproportionately motivated to partake in the research as they are the most interested and active students, who regularly follow seminars voluntarily. This may partly explain the absence of differences between the two groups of students. Another limitation regards the lack of some important information. Actually, anthropometric data of weight and height are self-reported and not measured, leading to under-reporting of weight while height was not indicated at all by the majority of the enrolled students. It is why the Body Mass Index was not included in our results. Analogously, information regarding family income, even if requested, was not supplied by respondents. Consequently, we could not assess the role of economic well-being in the relation between psychological well-being and the student's healthy behaviours. Taking the view of LM, further research is needed to investigate if the found positive association between healthy nutrition and the eudaimonic approach is still valid when considering the whole-food, plant-based diet.

Beyond these limits, this study contributes to LM research, as it suggests that healthy behaviour is easier to be acquired and maintained by individuals who aspire to achieve their life potential, and proposes the endowment of students in the healthcare area with the skills of counselling and well-being for a more effective spread of correct lifestyles in the general population. The protective relationship between positive psychological functioning and physical health was found, thus, promoting correct lifestyles can have an impact on health also through the enhancement of psychological well-being.

\section{Conclusions}

The main finding of our study was the positive relationship between virtuous lifestyle behaviours and psychological well-being. Considering terms of health promotion, the enhancement of correct lifestyles could lead to the identification of new methods aimed at preventing noncommunicable diseases and reducing the prevalence of behaviours at risk for health. The eudaimonic perspective and the Lifestyle Medicine approach imply a change of route for general practice and health promotion, from an approach based on deprivation and bans to an approach based on patient empowerment and self-management of their own lifestyle behaviours.

Author Contributions: Conceptualization: D.M. and S.M.; methodology: D.M., V.R., L.M., W.M. and S.M.; software: L.M. and F.B.; validation: D.M. and S.M.; formal analysis: D.M., V.R., L.M., W.M. and S.M.; investigation: F.B., G.P., G.L., V.G.; data curation: L.M. and F.B.; writing-original draft preparation: D.M., L.M. and S.M.; writing-review and editing: D.M., W.M. and S.M.; visualization: L.M. and F.B.; supervision: D.M. and S.M.; project administration: D.M. and S.M. All authors have read and agreed to the published version of the manuscript.

Funding: This research received no external funding.

Conflicts of Interest: The authors declare no conflict of interest.

\section{References}

1. World Health Organization. Global Status Report on Alcohol and Health. 2018. Available online: https: //www.who.int/substance_abuse/publications/global_alcohol_report/gsr_2018/en/ (accessed on 3 June 2020).

2. Centers for Disease Control and Prevention (CDC). National Center for Chronic Disease Prevention and Health Promotion. About Chronic Diseases. Available online: https://www.cdc.gov/chronicdisease/about/ index.htm\#risks (accessed on 3 June 2020). 
3. Lianov, L.; Johnson, M. Physician competencies for prescribing lifestyle medicine. JAMA 2010, 304, $202-203$. [CrossRef]

4. Nawaz, H.; Petraro, P.V.; Via, C.; Ullah, S.; Lim, L.; Wild, D.; Phillips, E.M. Lifestyle medicine curriculum for a preventive medicine residency program: Implementation and outcomes. Med. Educ. Online 2016, 21, 29339. [CrossRef]

5. Ockene, J.K.; Ashe, K.M.; Hayes, R.B.; Churchill, L.C.; Crawford, S.L.; Geller, A.C.; Ferguson, K.J. Design and rationale of the medical students learning weight management counseling skills (MSWeight) group randomized controlled trial. Contemp. Clin. Trials 2018, 64, 58-66. [CrossRef] [PubMed]

6. Lancaster, T.; Stead, L.F. Individual behavioural counselling for smoking cessation. Cochrane Database Syst. Rev. 2017, 3, 1-70. [CrossRef]

7. Chun, T.H.; Spirito, A.; Rakowski, W.; D'Onofrio, G.; Woolard, R.H. Beliefs and practices of pediatric emergency physicians and nurses regarding counseling alcohol-using adolescents: Can counseling practice be predicted? Pediatr. Emerg. Care 2011, 27, 812-825. [CrossRef]

8. Misuraca, R.; Miceli, S.; Teuscher, U. Three effective ways to nurture our brain: Physical activity, healthy nutrition, and music: A review. Eur. Psychol. 2017, 22, 101-120. [CrossRef]

9. Polak, R.; Pojednic, R.M.; Phillips, E.M. Lifestyle Medicine Education. Am. J. Lifestyle Med. 2015, 9, 361-367. [CrossRef] [PubMed]

10. Seligman, M.; Csikszentmihalyi, M. Positive psychology: An introduction. Am. Psychol. 2000, 55, 5-14. [CrossRef]

11. Lianov, L.S.; Fredrickson, B.L.; Barron, C.; Krishnaswami, J.; Wallace, A. Positive psychology in lifestyle medicine and health care: Strategies for implementation. Am. J. Lifestyle Med. 2019, 13, 480-486. [CrossRef]

12. Kiecolt-Glaser, J.K.; McGuire, L.; Robles, T.F.; Glaser, R. Emotions, morbidity, and mortality: New perspectives from psychoneuroimmunology. Annu. Rev. Psychol. 2002, 53, 83-107. [CrossRef]

13. Maniscalco, L.; Miceli, S.; Bono, F.; Matranga, D. Self-perceived health, objective health, and quality of life among people aged 50 and over: Interrelationship among health indicators in Italy, Spain, and Greece. Int. J. Environ. Res. Public Health 2020, 17, 2414. [CrossRef]

14. Huta, V.; Waterman, A.S. Eudaimonia and its distinction from hedonia: Developing a classification and terminology for understanding conceptual and operational definitions. J. Happiness Stud. 2014, 15, 1425-1456. [CrossRef]

15. Diener, E. Subjective well-being. In The Science of Well-Being: The Collected Works of Ed Diener; Springer: London, UK; New York, NY, USA, 2009; pp. 11-58.

16. Ryan, R.M.; Huta, V. Wellness as healthy functioning or wellness as happiness: The importance of Eudaimonic Thinking. J. Posit. Psychol. 2009, 4, 202-204. [CrossRef]

17. Huta, V. Eudaimonic and hedonic orientations: Theoretical considerations and research findings. In Handbook of Eudaimonic Well-Being; Vittersø, J., Ed.; Springer: Cham, Switzerland, 2016; pp. 215-231.

18. Dacey, M.; Arnstein, F.; Kennedy, M.A.; Wolfe, J.; Phillips, E.M. The impact of lifestyle medicine continuing education on provider knowledge, attitudes, and counseling behaviors. Med. Teach. 2013, 35, e1149-e1156. [CrossRef] [PubMed]

19. Trichopoulou, A.; Costacou, T.; Bamia, C.; Trichopoulos, D. Adherence to a Mediterranean diet and survival in a Greek population. N. Engl. J. Med. 2003, 348, 2599-2608. [CrossRef] [PubMed]

20. Willett, W.C.; Sacks, F.; Trichopoulou, A.; Drescher, G.; Ferro-Luzzi, A.; Helsing, E.; Trichopoulos, D. Mediterranean diet pyramid: A cultural model for healthy eating. Am. J. Clin. Nutr. 1995, 61, S1402-S1406. [CrossRef]

21. Kills Small, N.J.; Simons, J.S.; Stricherz, M. Assessing criterion validity of the Simple Screening Instrument for Alcohol and Other Drug Abuse (SSI-AOD) in a college population. Addict. Behav. 2007, 32, 2425-2431. [CrossRef]

22. Matranga, D.; Tabacchi, G.; Cangialosi, D. Sedentariness and weight status related to SES and family characteristics in Italian adults: Exploring geographic variability through multilevel models. Scand. J. Public Health 2018, 46, 548-556. [CrossRef]

23. Huta, V.; Ryan, R.M. Pursuing pleasure or virtue: The differential and overlapping well-being benefits of hedonic and eudaimonic motives. J. Happiness Stud. 2010, 11, 735-762. [CrossRef]

24. Prendergast, K.B.; Schofield, G.M.; Mackay, L.M. Associations between lifestyle behaviours and optimal wellbeing in a diverse sample of New Zealand adults. BMC Public Health 2016, 16, 62. [CrossRef] 
25. Castaldelli-Maia, J.M.; Ventriglio, A.; Bhugra, D. Tobacco smoking: From 'glamour'to 'stigma'. A comprehensive review. Psychiatry Clin. Neurosci. 2016, 70, 24-33. [CrossRef] [PubMed]

26. Costantino, C.; Maringhini, G.; Albeggiani, V.; Monte, C.; Lo Cascio, N.; Mazzucco, W. Perceived need for an international elective experience among Italian medical residents. Euromediterr. Biomed. J. 2013. [CrossRef]

27. Schwartz, S.J.; Waterman, A.S.; Vazsonyi, A.T.; Zamboanga, B.L.; Whitbourne, S.K.; Weisskirch, R.S.; Donnellan, M.B. The association of well-being with health risk behaviors in college-attending young adults. Appl. Dev. Sci. 2011, 15, 20-36. [CrossRef]

28. Frank, E.; Dresner, Y.; Shani, M.; Vinker, S. The association between physicians' and patients' preventive health practices. CMAJ 2013, 185, 649-653. [CrossRef] [PubMed]

29. Ryff, C.D. Psychological Well-Being Revisited: Advances in the Science and Practice of Eudaimonia. Psychother. Psychosom. 2014, 83, 10-28. [CrossRef]

30. Kimiecik, J. Exploring the promise of eudaimonic well-being within the practice of health promotion: The "how" is as important as the "what". J. Happiness Stud. 2011, 12, 769-792. [CrossRef]

(C) 2020 by the authors. Licensee MDPI, Basel, Switzerland. This article is an open access article distributed under the terms and conditions of the Creative Commons Attribution (CC BY) license (http://creativecommons.org/licenses/by/4.0/). 